This item was submitted to Loughborough's Research Repository by the author.

Items in Figshare are protected by copyright, with all rights reserved, unless otherwise indicated.

\title{
Phenomenological continuum theory of asphaltene-stabilized oil/water emulsions
}

PLEASE CITE THE PUBLISHED VERSION

https://doi.org/10.1021/acs.energyfuels.6b02430

\section{PUBLISHER}

(C) American Chemical Society (ACS)

\section{VERSION}

AM (Accepted Manuscript)

\section{PUBLISHER STATEMENT}

This work is made available according to the conditions of the Creative Commons Attribution-NonCommercialNoDerivatives 4.0 International (CC BY-NC-ND 4.0) licence. Full details of this licence are available at: https://creativecommons.org/licenses/by-nc-nd/4.0/

\section{LICENCE}

CC BY-NC-ND 4.0

\section{REPOSITORY RECORD}

Toth, Gyula, Juri Selvag, and Bjorn Kvamme. 2019. "Phenomenological Continuum Theory of Asphaltenestabilized Oil/water Emulsions". figshare. https://hdl.handle.net/2134/27270. 


\title{
A phenomenological continuum theory of asphaltene stabilized oil/water emulsions
}

\author{
Gyula I. Tóth, ${ }^{*, \dagger, \ddagger}$ Juri Selvåg, ${ }^{\dagger}$ and Bjørn Kvamme ${ }^{\dagger}$ \\ Department of Physics and Technology, University of Bergen, Allégaten 55, 5007 Bergen, \\ Norway, and Institute for Solid State Physics and Optics, Wigner Research Centre for \\ Physics, P.O.Box 49, H-1525 Budapest, Hungary \\ E-mail: gyula.toth@ift.uib.no
}

\begin{abstract}
In this paper we use a phenomenological continuum theory of the Ginzburg-Landau type to address emulsion formation in water/light hydrocarbon/asphaltene systems. Based on the results of recent molecular dynamics simulations, we first calibrate the model parameters and show, that the theory produces a reasonable equation of state. Next, the coalescence of oil droplets is studied by a convection-diffusion dynamics as a function of both the surface coverage and the viscosity contrast between the asphaltene and the bulk liquids. We show, that, besides the traditional thermodynamic interpretation of emulsion formation, the timescale of drop coalescence can be controlled independently from the interfacial tension drop, which offers an alternative, solely kinetic driven mechanism of emulsion formation.
\end{abstract}

\footnotetext{
*To whom correspondence should be addressed

$\dagger$ University of Bergen

${ }^{\ddagger}$ Wigner Research Centre for Physics
} 


\section{Introduction}

Emulsions are known as mechanically stable mixtures of at least two, immiscible liquids, which is due to the presence of a foreign material, called surfactant. ${ }^{1}$ Emulsions appear in many fields of everyday life, ranging from cosmetics and food processing, ${ }^{2}$ to medical applications, ${ }^{3-5}$ or even enhanced crude oil recovery. ${ }^{6,7}$ Regarding the latter, asphaltene stabilized systems are surrounded by constantly increasing interest for two reasons: (i) separation of water containing crude oil emulsions is a complex process, ${ }^{8}$ and (ii) understanding the microscopic mechanisms involved in emulsion formation in these systems might help in designing new generation of surfactants, ${ }^{9,10}$ which would open the possibility of developing a new, environmentally sound and efficient technique for combined $\mathrm{CO}_{2}$ sequestration and Enhanced Oil Recovery.

In the traditional interpretation, emulsions forms by thermodynamic reason. The molecules of the surfactant migrate to the liquid-liquid interfaces and lower the interfacial tension. The interfacial tension is a monotonously decreasing function of the surfactant concentration, and tends to zero. Since the thermodynamic driving force of the phase separation is the interfacial tension, the phase separation process practically stops at vanishing interfacial tension, and the mixture is stabilized. Although this is a simple and generally accepted explanation, this is not the only possible mechanism of emulsion formation (at least theoretically). The general requirement for emulsion formation is the termination of the phase separation process. Besides the thermodynamic driving force (i.e. the interfacial tension), the process speed also depends on kinetic factors, therefore, the phase separation also stops for diverging viscosity and vanishing diffusion coeffients, independently from the interfacial tension drop. This might happen in asphaltene containing oil/water systems: Asphaltenes are solid in the bulk, and, according to the results of molecular dynamic simulations, ${ }^{11}$ even monolayers forming at oil/water interfaces behave like the bulk solid. In this case, the fluid flow is locally suppressed (because of the diverging viscosity in the solid layer), and the deformation of the solid layer is possible only by diffusion, which is usually slower by at least 3 orders of magni- 
tude than convective transport. If the diffusion of liquid molecules is also suppressed in the surfactant layer, phase separation practically stops, since the transport of liquid molecules accross the interface is forbidden. The macroscopic picture of this scenario is simple: When oil-filled ping-pong balls floating in water meet, no drop coalescence happens, since the thin solid plastic shell completely blocks the transport of oil and water. The question is, whether this scenario is adequate on the nanoscale. If it is, it can reveal the mechanism of emulsion formation in asphaltene containing oil/water systems, in which the interfacial tension drop is found to be strongly limited $(\lesssim 50 \%),{ }^{12-14}$ but the emulsion still forms. ${ }^{15,16}$

In measuring the interfacial tension, atomistic approaches can be used to model equilibrium interfaces, and the results are in good agreement with experimental data. ${ }^{11} \mathrm{Nev-}$ ertheless, the simulations are very limited in both space and time, thus necessitating the development of a mesocale theory capable of addressing the emulsion formation process itself. Continuum theories describing a surfactant coated interfaces originate from Gompper and Zschocke, ${ }^{17}$ and Theissen and Gompper, ${ }^{18}$ while the model of Teramoto and Yonezawa ${ }^{19}$ has been used to describe droplet growth dynamics in the oil/water system. A most successful version of the theory is developed by van der Sman and van der Graaf, ${ }^{20-22}$ which was generalized by Liu and Zhang. ${ }^{23}$ A similar approach was published by Teng, Chern and Lai. ${ }^{24}$ The models have been applied for addressing the Marangoni effect appearing in these systems due to the inhomogeneous interfacial tension. A comparative study of the aforementioned models is available by $\mathrm{Li}$ and $\mathrm{Kim},{ }^{25}$ while a detailed numerical analysis was done by S. Engblom and co-workers. ${ }^{26}$ Theoretical analyses and consistent formulation of the generalized van der Sman / van der Graaf model for two qualitatively different systems, as well as numerical simulations of spinodal decomposition in diffusion driven systems have recently been published by Tóth and Kvamme. ${ }^{9,10}$ All the aforementioned works addressed thermodynamics driven emulsion formation in liquid systems, i.e. a situation, in which the phase separation stops because of the vanishing interfacial tension. Although some works include hydrodynamics, while others do not, the phase separation speed is expected to be essentially 
proportional to the interfacial tension, since constant kinetic parameters (viscosity and diffusion coefficients) were used in these works. In contrast, a recent study of Tóth and Kvamme ${ }^{10}$ addressed surfactant assisted liquid phase separation in water/heptane/asphaltene systems by a convection-diffusion dynamics with local composition dependent viscosity. According to the results, a composition dependent viscosity may have a major effect on the speed of phase separation. Therefore, in the present work we study the process of drop coalescence as a function of both the surface coverage and the viscosity of the asphaltene to check whether the concept of dynamic emulsion formation is relevant for these systems.

\section{Theory}

In the Ginzburg-Landau type approach of the van der Sman and van der Graaf, ${ }^{20}$ the free energy $F$ of the inhomogeneous system is given in terms of the two conserved order parameters: the difference of the local and temporal mass densities of the liquids normalized by their equilibrium values, i.e. $\phi(\mathbf{r}, t):=\left[\rho_{2}(\mathbf{r}, t) / \rho_{2}^{0}\right]-\left[\rho_{1}(\mathbf{r}, t) / \rho_{1}^{0}\right]$, and the normalized surfactant mass density $\psi(\mathbf{r}, t):=\rho_{3}(\mathbf{r}, t) / \rho_{3}^{0}$. The dimensionless phenomenological free energy functional reads: ${ }^{9,10,20,26}$

$$
\mathcal{F}=\int d \mathbf{r}\left\{f_{C H}(\phi)+f_{\psi}(\psi)+f_{1}(\phi, \psi)\right\}
$$

where

$$
\begin{aligned}
f_{C H}(\phi) & =g(\phi)+(\nabla \phi)^{2} ; \\
f_{\psi}(\psi) & =\frac{1}{\beta}[\psi \log (\psi)+(1-\psi) \log (1-\psi)]+\gamma(\nabla \psi)^{2} ; \\
f_{1}(\psi, \phi) & =\alpha \psi f_{c}(\phi) .
\end{aligned}
$$

Here $f_{C H}(\phi)$ is the usual Cahn-Hilliard free energy density of a phase separating binary system, where $g(\phi)=\frac{\left(1-\phi^{2}\right)^{2}}{4}$. The logarithmic term in $f_{\psi}(\psi)$ is the entropic part of the free 
energy of mixing of the surfactant with the bulk phase, while the square gradient term is the lowest order contribution to the energy accounting for spatial correlations in interacting systems. ${ }^{27,28}$ In general, the free energy density should contain all $\nabla c_{i} \cdot \nabla c_{j}$ terms in an $N$-component systems characterized by the compositions $\left\{c_{1}, c_{2}, \ldots, c_{N}\right\}$, however these terms are neglected here. Finally, $f_{c}(\phi)=f_{e}(\phi)-\delta_{S}(\phi)$ linearly couples the binary phase separation to the surfactant field. The enthalpic contribution $f_{e}(\phi)=\phi^{2} / 2$ expresses that presence of the surfactant is energetically penalized in the bulk liquids, while the surface delta function $\delta_{S}(\phi)$ can be regularized on several fashions for continuum models. ${ }^{26}$ In agreement with the results of our previous works ${ }^{9}$ we choose $\delta_{S}(\phi)=g(\phi)$ resulting in broadening interface with increasing surfactant composition. In the surfactant-free case (i.e. $\psi(\mathbf{r}, t) \equiv 0)$, Eq. (1) reduces to a simple Cahn-Hilliard model generating the equilibrium planar interface solution $\phi_{0}(x)=\tanh (x / 2)$. Accordingly, the length scale $\lambda=\delta / 2$ and the free energy density scale $f_{0}=(3 / 2)(\sigma / \delta)$ results in the dimensional free energy $F=F_{0} \mathcal{F}$, where $F_{0}=(3 / 2)(\delta / 2)^{D}(\sigma / \delta)$ (here $D$ is the spatial dimensionality of the problem), generating thus the surfactant-free planar interface solution $\phi_{0}(z)=\tanh (z / \delta)$ with interfacial tension $\sigma$. The parameters $\alpha, \beta$ and $\gamma$ can be interpreted as follows: $\alpha$ is a coupling parameter expressing that the surfactant wants to migrate from the bulk liquids $(\phi= \pm 1)$ to the interface $(\phi=0)$, while $\beta$ is necessary to establish (Langmuir or Frumkin type) physical adsorption isotherms. ${ }^{9}$ Furthermore, $\gamma$ controls the expansion of the surfactant at the interfaces. It is worth mentioning, that additional terms mimicking interactions related to the presence of the surfactant ${ }^{29}$ are also can be included in the free energy functional. While some of the possible extra terms (like adding a term $\propto \psi^{2}$ accounting for the interactions between surfactant layers) make no qualitative difference in the behavior of the model, including more complex interactions do: For instance, modeling steric interactions necessitate the introduction of the geometry of the surfactant molecules, which can be done by re-formulating Eq. (1) in terms of a vector phase-field $\vec{\psi}$. New terms in Eq. (1) then can be included expressing that (i) the orientation of the surfactant molecules $\vec{n}_{\psi}=\vec{\psi} /|\vec{\psi}|$ must mach with the normal 
of liquid-liquid interfaces $\vec{n}_{\phi}=\nabla \phi /|\nabla \phi|$, and that (ii) the spatial change of $\vec{n}_{\psi}$ is penalized. Such a description can be done on the basis of orientation / vector field based phase-field descriptions, ${ }^{30}$ or alternatively, multidomain-field models. ${ }^{31}$

The general planar (1-dimensional) equilibrium solution of Eq. (1) can be determined by solving the Euler-Lagrange equations $\delta \mathcal{F} / \delta \phi=0$ and $\delta \mathcal{F} / \delta \psi=\mu$, where $\delta / \delta y$ stands for the functional derivative of $\mathcal{F}$ with respect to $y=\phi, \psi$, i.e.:

$$
\begin{aligned}
& \frac{\delta \mathcal{F}}{\delta \phi}=(1-\alpha \psi)\left(\phi^{3}-\phi\right)+\alpha \psi \phi-2\left(\nabla^{2} \phi\right) \\
& \frac{\delta \mathcal{F}}{\delta \psi}=-\alpha g(\phi)+\alpha \frac{\phi^{2}}{2}+\frac{1}{\beta} \log \left(\frac{\psi}{1-\psi}\right)-2 \gamma\left(\nabla^{2} \psi\right)
\end{aligned}
$$

while $\mu=(\delta \mathcal{F} / \delta \psi)_{\psi_{0}}$ is the equilibrium diffusion potential of the surfactant in the bulk liquids, i.e. far from the interface. We note, that the value of $\phi_{0}$ emerges from the symmetry properties of the Euler-Lagrange equations and is related to $\psi_{0}$ as

$$
\phi_{0}^{2}=\frac{1-2 \alpha \psi_{0}}{1-\alpha \psi_{0}}
$$

The time evolution of the system is driven by a convection-diffusion type dynamics. Assuming incompressible flow, the scaled kinetic equations read as: ${ }^{10}$

$$
\begin{aligned}
\dot{\phi} & =\nabla^{2}(\delta \mathcal{F} / \delta \phi) \\
\tau \dot{\psi} & =\nabla \cdot[\psi(1-\psi) \nabla(\delta \mathcal{F} / \delta \psi)] \\
\dot{\mathbf{v}} & =\nabla \cdot(\mathbb{R}+\mathbb{D}) \\
\nabla \cdot \mathbf{v} & =0
\end{aligned}
$$

where $\mathbf{v}(\mathbf{r}, t)$ is the macroscopic velocity field, $\dot{y}=\partial y / \partial t+\mathbf{v} \cdot \nabla y$ denotes the material derivative, $\tau$ is the relative time scale of the surfactant diffusion, while the reversible and 
viscous stress tensors read as:

$$
\begin{aligned}
& \mathbb{R}=-p \mathbb{I}-w[(\nabla \phi \otimes \nabla \phi)+\gamma(\nabla \psi \otimes \nabla \psi)] \\
& \mathbb{D}=\nu h(\phi, \psi)\left[(\nabla \otimes \mathbf{v})+(\nabla \otimes \mathbf{v})^{T}\right],
\end{aligned}
$$

where $p$ is the non-equilibrium thermodynamic pressure, $w$ emerges from the scaling, and $\nu$ is the dimensionless average viscosity of the liquids. We note, that the form of the non-classical reversible stress $\mathbb{R}$ defined by Eq. (9) emerges from the least action principle, ${ }^{32}$ yielding the Gibbs-Duhem relation :

$$
\nabla \cdot \mathbb{R}=-\phi \nabla \frac{\delta \mathcal{F}}{\delta \phi}-\psi \nabla \frac{\delta \mathcal{F}}{\delta \psi}
$$

In order to model the solid-like behavior of the bulk surfactant, we use

$$
h(\phi, \psi):=\frac{1-\phi}{2} x_{1}+\frac{1+\phi}{2} x_{2}+A[\exp (b \psi)-1]
$$

where $x_{1}$ and $x_{2}$ are the viscosities of the liquids relative to the average liquid viscosity, while $A$ and $b$ are experimental constants accounting for the presence of the asphaltene. As indicated by Table 1, the time scale $\epsilon$ and the dynamic parameters $\tau, w$ and $\nu$ can be expressed in terms of physical quantities, such as the equilibrium interfacial tension $(\sigma)$ and interface thickness $(\delta)$, the liquid-liquid and the surfactant diffusion constants $\left(D_{0}\right.$ and $D_{\psi}$, respectively), the average mass density of the system $(\rho)$, and the average dynamic viscosity of the liquids $(\mu)$.

From the viewpoint of physical consistency, it is important to justify the assumption of incompressibility. In case of constant total density $\left(\rho_{0}\right)$, the local volume fraction $\left(\varphi_{i}=\right.$ $\left.d V_{i} / d V\right)$ and mass fraction $\left(c_{i}=d m_{i} / d m\right)$ of a component coincide $\left(c_{i}=\varphi_{i}=\rho_{i} / \rho_{0}\right.$, where $\rho_{i}=d m_{i} / d V$ is the local mass density of the component), ${ }^{33-35}$ and can be related to the 
variables of the model as:

$$
\begin{aligned}
& \varphi_{1}(\mathbf{r}, t)=[1-\phi(\mathbf{r}, t)-\psi(\mathbf{r}, t)] / 2 ; \\
& \varphi_{2}(\mathbf{r}, t)=[1+\phi(\mathbf{r}, t)-\psi(\mathbf{r}, t)] / 2 ; \\
& \varphi_{3}(\mathbf{r}, t)=\psi(\mathbf{r}, t) .
\end{aligned}
$$

Consequently, the physical interpretation of $\phi(\mathbf{r}, t)$ and $\psi(\mathbf{r}, t)$ is recovered via $\left[\rho_{2}(\mathbf{r}, t) / \rho_{2}^{0}\right]-$ $\left[\rho_{1}(\mathbf{r}, t) / \rho_{1}^{0}\right]=\varphi_{2}(\mathbf{r}, t)-\varphi_{1}(\mathbf{r}, t)=\phi(\mathbf{r}, t)$ and $\rho_{3}(\mathbf{r}, t) / \rho_{3}^{0}=\varphi_{3}(\mathbf{r}, t)=\psi(\mathbf{r}, t)$. Moreover, the conservation of the component densities indicate the conservation of $\phi(\mathbf{r}, t)$ and $\psi(\mathbf{r}, t)$. Finally, the incompressibility is also satisfied, since:

$$
\sum_{i=1}^{3} \rho_{i}(\mathbf{r}, t)=\rho_{0} \sum_{i=1}^{3} \varphi(\mathbf{r}, t) \equiv \rho_{0},
$$

independently from the value of $\phi(\mathbf{r}, t)$ and $\psi(\mathbf{r}, t)$.

Table 1: Parameters of the dynamic equation. See the main text for details.

\begin{tabular}{cccc}
\hline$\epsilon$ & $\tau$ & $w$ & $\nu$ \\
\hline$\frac{\delta^{2}}{2 D_{0}^{2}}$ & $\frac{1}{2 \beta} \frac{D_{0}}{D_{\psi}}$ & $\frac{3 \sigma \delta}{D_{0}^{2} \rho}$ & $\frac{\mu}{\rho} \frac{2}{D_{0}}$
\end{tabular}

\section{Results}

\section{Calibration}

In order to calibrate the theory, the equation of state (EOS) has to be determined numerically. The EOS is defined as the interfacial tension (relative to the surfactant-free system) as a function of the surface coverage. The relative interfacial tension $(\kappa)$ and the scaled surface 
coverage $(\omega)$ read:

$$
\begin{aligned}
\kappa & =\frac{3}{4} \int_{-\infty}^{+\infty} d x\left\{I\left[\phi^{*}(x), \psi^{*}(x)\right]-I\left[\phi_{0}, \psi_{0}\right]-\mu\left[\psi^{*}(x)-\psi_{0}\right]\right\} \\
\omega & =\int_{-\infty}^{+\infty} d x\left\{\psi^{*}(x)-\psi_{0}\right\},
\end{aligned}
$$

where $I[$.$] stands for the total integrand of Eq. (1), \phi^{*}(x)$ and $\psi^{*}(x)$ denote the solution of the Euler-Lagrange equations for the bulk surfactant load $\psi_{0}$ [the corresponding $\phi_{0}$ is defined by Eq. (4)]. For a schematic 1-dimensional solution of the EL equations see Fig 1. The

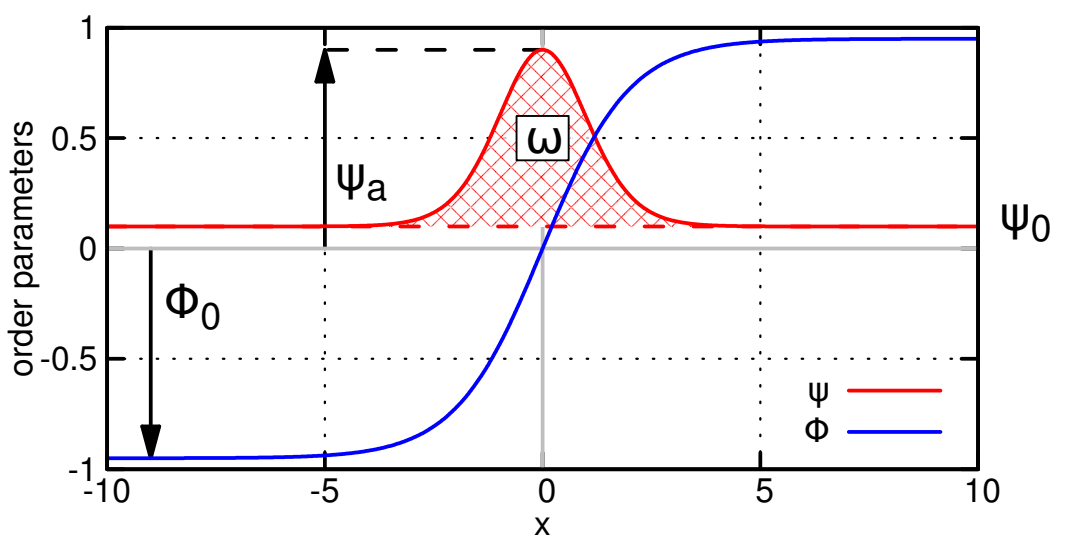

Figure 1: Schematic equilibrium planar interface solution of the model. The liquid-liquid interface is represented by a $-1 \rightarrow+1$ transition in the liquid-liquid order parameter $\phi$ (solid blue curve), while the surfactant composition $\psi$ shows a maximum at the interface (red curve). The scenario is characterized by the bulk liquid density $\left(\phi_{0}\right)$ and surfactant composition $\left(\psi_{0}\right)$, the surface load $\psi_{a}$, and/or the total amount of absorbed surfactant per unit area, the surface coverage $(\omega)$.

dimensional surface coverage $\left(\Gamma\right.$, measured in molecules $\left./ \mathrm{nm}^{2}\right)$ can be calculated as

$$
\Gamma=\bar{n}(\delta / 2) w
$$

where $\bar{n}$ is the average molar density of asphaltenes (in the bulk state), and $\delta$ the interface width of the surfactant-free system. For the Nexbase 2002 water/hydrocarbon/asphaltene 
system the EOS is known from experiments, indicating the Langmuir form: ${ }^{14}$

$$
\kappa(\Gamma)=1+\left(\frac{R T}{\sigma}\right) \Gamma_{\infty} \log \left(1-\frac{\Gamma}{\Gamma_{\infty}}\right)
$$

where $\sigma_{0}$ is the interfacial tension in the surfactant-free system, $R=8.31 \mathrm{~J} /(\mathrm{mol} \mathrm{K})$ the gas constant and $T$ the temperature. Fig. 2 shows the experimental equation of state for $T=300 \mathrm{~K}, \sigma=40 \mathrm{~mJ} / \mathrm{m}^{2}$ and $\Gamma_{\infty}=3$ molecules $/ \mathrm{nm}^{2},{ }^{14}$ corresponding to $\omega_{\infty}=20$ for $\delta=2.5 \AA$ (indicating $\approx 0.5 \mathrm{~nm} 10 \%-90 \%$ interface width ${ }^{11}$ ) and $\bar{n}=2000 \mathrm{~mol} / \mathrm{m}^{3}$. To find the best fitting model EOS, we solved the 1-dimensional diffusion equations described by Eqns. (5)-(6) for $\mathbf{v} \equiv 0$ as a function of the model parameters $(\alpha, \beta, \gamma)$ and the surface coverage $\omega$. The reason is that the dynamic equations prescribe conserved variables, i.e. the total amount of the surfactant is fixed in a calculation. At fixed values of the model parameters the dynamic equations were solved on an $N=4096$ pixel 1-dimensional grid with grid spacing $h=0.1$ for 128 different surfactant loads simultaneously on one GPU (Graphical Processing Unit) by using a quasi-spectral, semi-implicit operator splitting based numerical scheme, ${ }^{36,37}$ indicating thus periodic boundary conditions. The initial conditions for the different surfactant loads read $\phi(x, 0)=\{1-\tanh [(x-x 0) / 2]\}\{1+\tanh [(x+x 0) / 2]\} / 2-1$ and $\psi(x, 0)=2 \Omega_{i} / L, i=1 \ldots 250$. Here $L=N h$ and $x_{0}=L / 4$, while the spatial coordinate for grid index $j=0 \ldots N-1$ is calculated as $x=j h-L / 2$. Note that for almost perfectly absorbing interfaces $\Omega_{i} \approx \omega_{i}$, i.e. the surface coverage should be equal to the total amount of surfactant. After 25 million time steps with $d t=0.1$, the maximal relative change in the bulk surfactant load / surface coverage / interfacial tension was less than $10^{-7}$ in 100.000 time steps. The convergent numerical equation of state $\kappa(\omega)$ for $\alpha=0.625, \beta=32$ and $\gamma=0.25$ is shown in Fig 2, which indicates a reasonable fit to the experimental curve in the following sense: The experimental curve ends at a certain interface coverage, since in most cases only monolayers of big surfactant molecules can form at an interface, therefore, the interface coverage has a certain limit. Beyond this limit, the extra surfactant molecules 


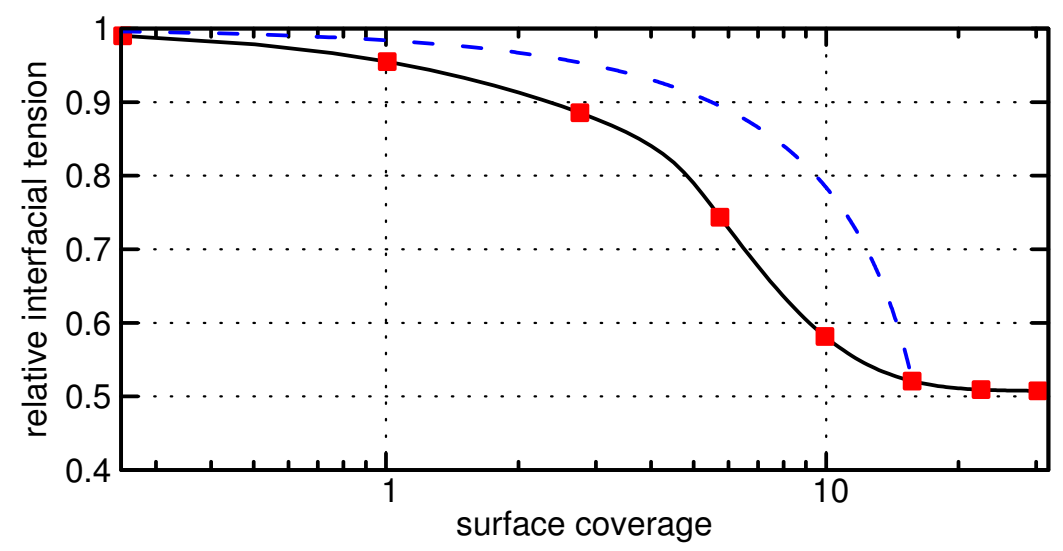

Figure 2: Relative interfacial tension drop [equation of state (EOS)] in the present model (solid curve) as a function of the surface coverage $\omega$. Red squares denote the location of the numerical simulations for the drop coalescence study. For comparison, an experimental EOS is also shown (dashed curve).

remain in the bulk phases, and often form micelles beyond the solubility limit. This limit is, however, not included in our model, i.e. the interfaces can be infinitely loaded. Nevertheless, the interfacial tension saturates for large loads, therefore, similarly to the experimental results, the relative interfacial tension cannot be less than 0.5 , and the thermodynamics driven emulion formation (attributed to vanishing interfacial tension) is excluded. Since the slowdown of the drop coalescence is a qualitative effect (i.e. the drop colascence speed must decrease by orders of magnitude), the model EOS together with the phenomenologically contructed viscosity should be suitable to address the phenomenon, despite the lack of quantitativeness. Fig 3 shows order parameter profiles for surface coverage $\omega=24$, which was a fitting point to molecular dynamics data ${ }^{11}$ (for the water/heptane/asphaltene model system) in our previous study. (In the MD simulations $\omega=24$ was possible to reach, since it was a slightly different model system compared to the experimental one.) Since the figure shows a reasonable agreement between the results of molecular dynamics data and our numerical order parameter profiles, indicating thus the physically correct calibration of highly loaded interfaces. We note, that our goal is not to choose a specific material but to describe the characteristic properties of water/light hydrocarbon/asphaltene systems. 


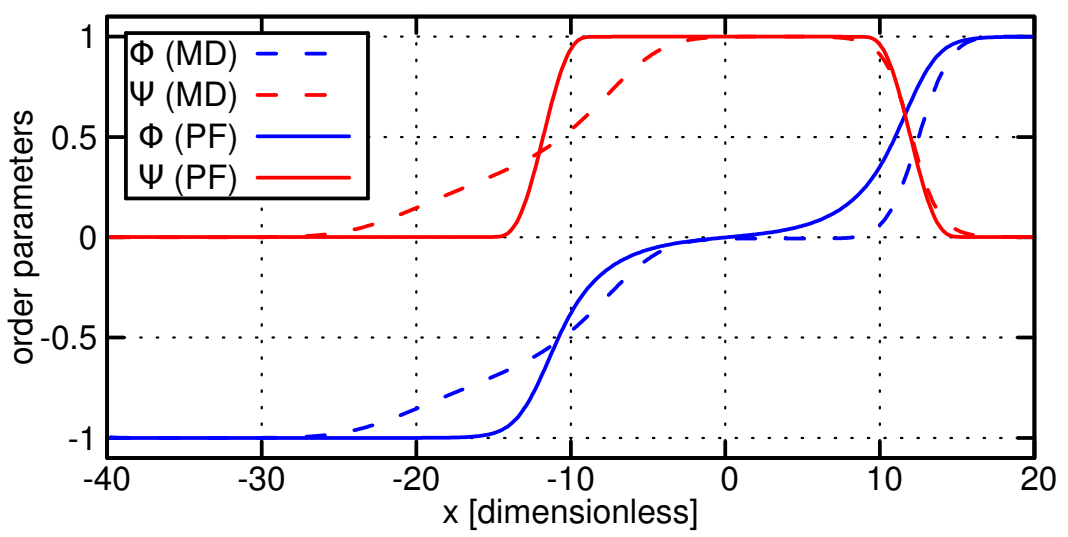

Figure 3: Order parameter profiles for the highly loaded equilibrium planar interface from molecular dynamics simulations (dashed curves) and from the present model (solid curves) for surface coverage $\omega=24$.

The parameters of the dynamic equations were chosen to coincide with the parameters in our previous study. Therefore, typical values mimicking a water/heptane/asphaltene system were used: At reservoir circumstances (room temperature, $T \approx 300 K$, and $10-100 \mathrm{MPa}$ pressure) the mass average mass density of the constituents reads as $\rho_{w} \approx 850 \mathrm{~kg} / \mathrm{m}^{3} .{ }^{11}$ The diffusion constant for the heptane in water is approximately $D_{0} \approx 7.0 \times 10^{-10} \mathrm{~m}^{2} / \mathrm{s},{ }^{38}$ while the typical diffusion constant of the asphaltene in heptane reads as $D_{\psi} \approx 1.5 \times 10^{-10}$ $\mathrm{m}^{2} / \mathrm{s}$. ${ }^{39}$ The kinematic viscosities read as $\mu_{w}^{0}=1 \mathrm{mPas}^{40}$ and $\mu_{h}^{0}=0.4 \mathrm{mPas}^{41}$ for the pure water and heptane, respectively. The viscosity of the heptane/asphaltene system can be approximated by Eq. (12), resulting in $b \approx 25.75,{ }^{41}$ while $A$ is to be determined according to the desired viscosity contrasts in the simulations. finally, the physical parameters result in the model parameters $\tilde{\tau}_{\psi}=0.073, \tilde{w}=1.73 \times 10^{4}$, whereas the dimensionless viscosities $\operatorname{read}$ as $\tilde{\nu}_{w}=2857$ and $\tilde{\nu}_{h}=1633$.

\section{Drop coalescence study}

After fitting the model parameters resulting in a reasonable equation of state and order parameter profiles, drop coalescence has been studied as a function of the surface coverage and the viscosity contrast between the bulk surfactant and the liquids. Our previous study 
predicted significant hydrodynamic effect even for small surface coverage during the eraly stage of phase separation, therefore, we investigated the speed of drop coalescence in an already developed emulsion. As a first step, a circular droplet was equilibrated for a fixed surface coverage by solving the dynamic equations. The equations have been solved for $\mathbf{v} \equiv 0$ on a $2048 \times 2048$ domain with grid spacing $h=0.5$ and $\delta t=0.1$ time step. The physical diameter of a droplet was then $d \approx 0.1 \mu \mathrm{m}$. The initial condition was interpolated by using the 1-dimensional planar interface solution. After 1 million time steps the droplet was equilibrated. As the initial condition of the drop coalescence calculations, we put 2 equilibrated droplets from the previous calculation next to each other with a slight $(\approx 10 \%$ corresponding to $\psi=0.1$ ) overlap in the surfactant fields (see Fig 4), thus mimicking the event when two droplets meet. The drop coalescence simulations were performed on a $4096 \times 4096$ grid with
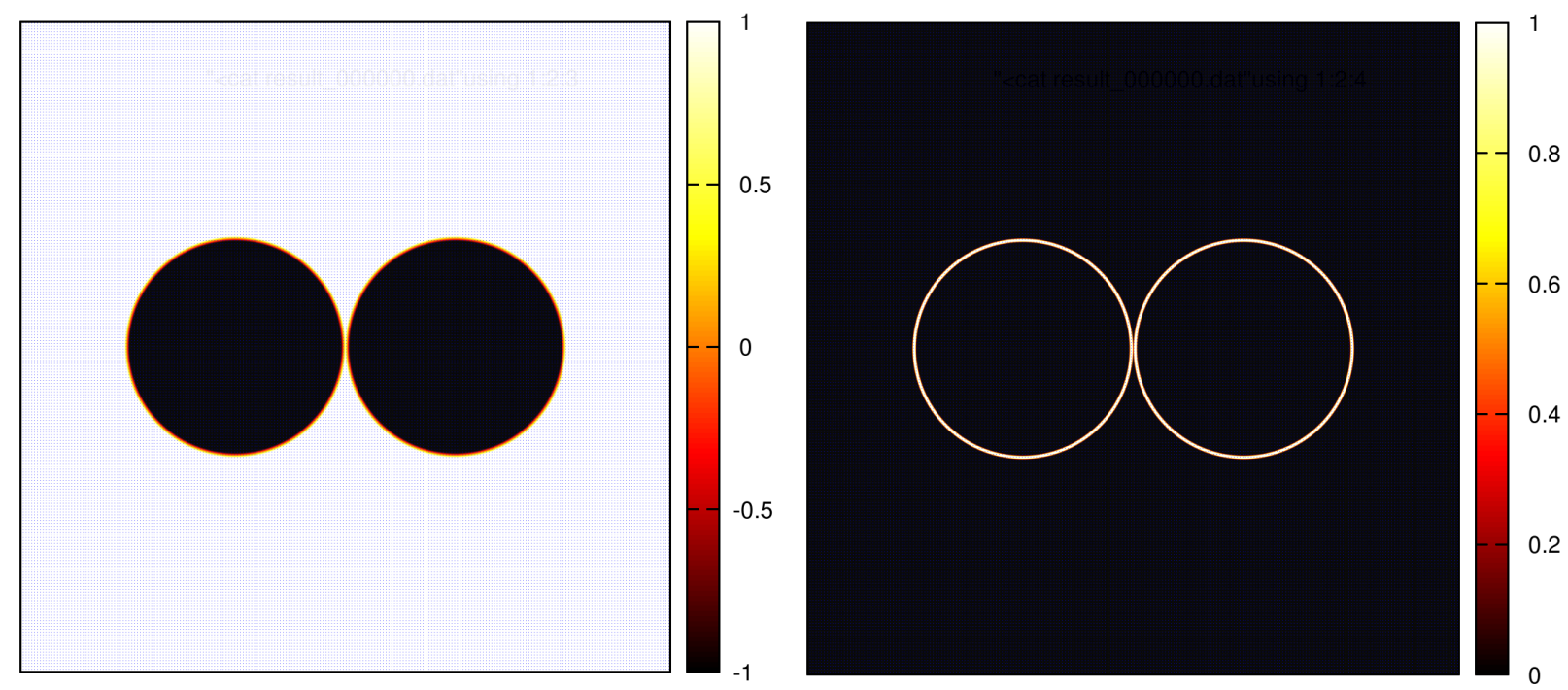

Figure 4: Initial condition for the drop coalescence simulations: liquid-liquid order parameter $(\phi(\mathbf{r}, 0)$, on the left), and surfactant concentration $(\psi(, 0)$, on the right). Two equilibrated oil droplets at a certain surface coverage are placed into the water next to each other. Note that only the surfactant layers are in contact with each other (on the right). Correspondingly, a thin water layer can be observed between the two oil droplets (on the left).

grid spacing $h=0.5$ and $d t=2.5 \times 10^{-3}$ in the presence of fluid flow. The phenomenon was studied as the function of both the surface coverage and the viscosity contrast between the bulk surfactant and the liquids (see Eq. 12). After starting a simulation, fluid flow was 
generated at the liquid-liquid interfaces (due to the capillary stress), initiating thus the drop coalescence process. To quantify the speed of the process, we detected $r(t)$, the position of the $\phi(\mathbf{r}, t)=0$ contour line along the vertical axis (see Fig 5 ) as a function of time. Naturally,
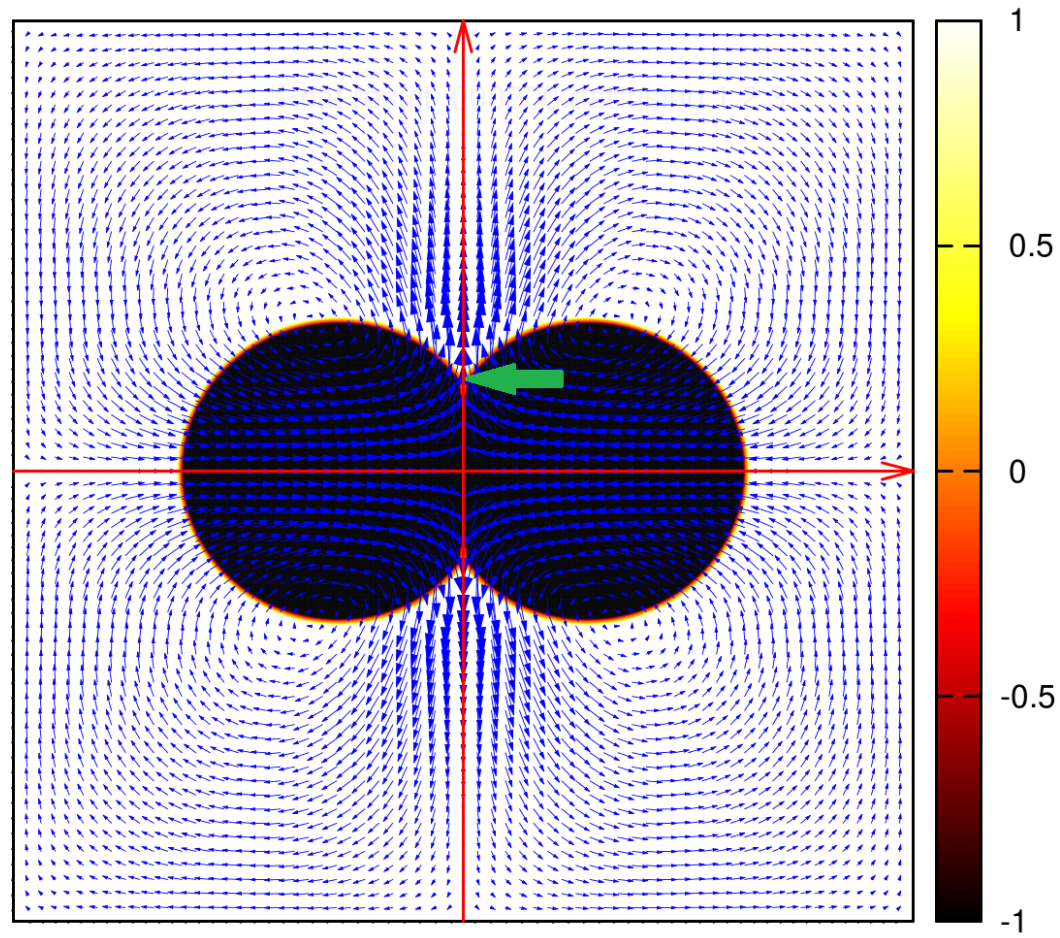

Figure 5: Snapshot of the liquid-liquid order parameter $\phi(\mathbf{r}, t)$ during a drop coalescence simulation. To describe the process, the position of the $\phi(\mathbf{r}, t)=0$ isoline along the vertical axis (indicated by the green arrow in the figure) is tracked as a function of time. The velocity field is indicated by blue vectors.

no such point exists at $t=0$ in the present of surfactant, since the two droplets are separated by a thin liquid layer: Note, that the surfactant layers are in contact in the initial condition, not the $\phi=0$ contour lines of the two droplets (except the surfactant free case). Therefore, a transient time $t_{0}$ is needed until this point appears $\left[t_{0} \approx 250\right.$ in Fig $\left.6(\mathrm{a})\right]$. The front position $r(t)$ then has two characteristic regimes: (i) a quasi-linear developing period between $t_{0}$ and $t_{1}=t\left(r=r_{1}\right)$, where $r_{1} \approx r_{0} / 3$, and $r_{0}$ is the final radius of the coalesced droplet, and (ii) the saturation regime for $t>t_{1}$. To measure the speed of drop coalescence we used two definitions [see Fig 6(b)]: (i) we measured the total elapsed time $\tau$ until the front position reaches a particular value $r_{1}$, and (ii) we also measured $\Delta \tau$, the time elapsed 
between the two front positions $r_{1} \pm \Delta r$. The only important thing is that all the front positions should be in the quasi-linear developing stage, since in the long-time regime $r(t)$ converges to the same value, $r_{0}$, independently from the parameters. Accordingly, we chose $r_{1}:=150 \approx r_{0} / 3$, and $\Delta r=50$, and evaluated $\tau$ and $\Delta t$ as a function of both the surface coverage and the viscosity contrast. The simulations were performed for surface coverages $\omega \approx 0,0.75,2.5,5.5,9.7,15.5,22$ and 30 (shown in Fig 2 ), while the values $A=1.5 \times 10^{-8+p}$ ( $p=1,2,3)$ were used in Eq. (12) indicating thus bulk surfactant / bulk liquid viscosity contrasts $10^{1}, 10^{2}$, and $10^{3}$, respectively. The main results of the present work are summa-
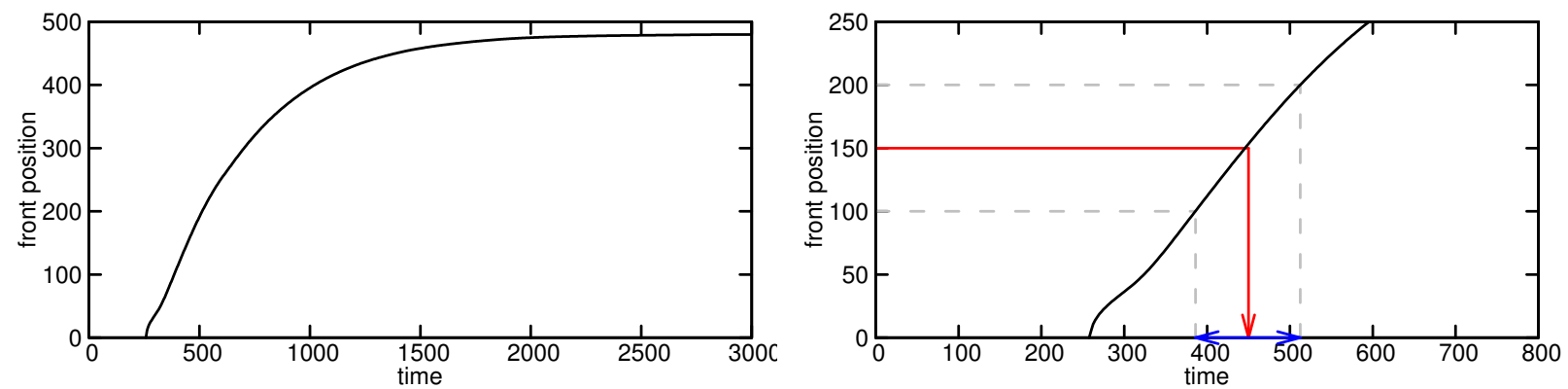

Figure 6: Time dependence of the front position $[r(t)$, see Fig 5 for the definition] during drop coalescence. The speed of coalescence is quantified by (i) the total elapsed time $(\tau)$ from the beginning of the simulation, until the front position reaches a certain value (shown by the red arrow), or (ii) the elapsed time between two front positions $(\Delta \tau$, indicated by the blue arrow).

rized in Fig. 7, where the relative elapsed time $s(\omega):=\tau_{0} / \tau(\omega)\left[\right.$ and $\left.\Delta \tau_{0} / \Delta \tau(\omega)\right]$ is shown for different viscosity contrasts. Here $\tau_{0}$ (and $\Delta \tau_{0}$ ) is the elapsed time in the surfactant free system, therefore, the relative elapsed time shows the deceleration factor of the process. As expected, all curves start at $s(0)=1$ (surfactant-free system), and show decreasing tendency with increasing surface coverage, meaning that the presence of the surfactant decelerates the coalescence. The same is can be observed as a function of the viscosity contrast: increasing viscosity contrast results in decreasing process speed. The most interesting part is, that at high interface loads $(\omega \approx 20-30$, while $\omega=24$ is the fitting for the highly loaded profile, see Fig 3) the process slows down enormously, i.e. by orders of magnitude (note the logscale on Fig 7). Roughly speaking, the slowdown of the coalescence process is inversely 

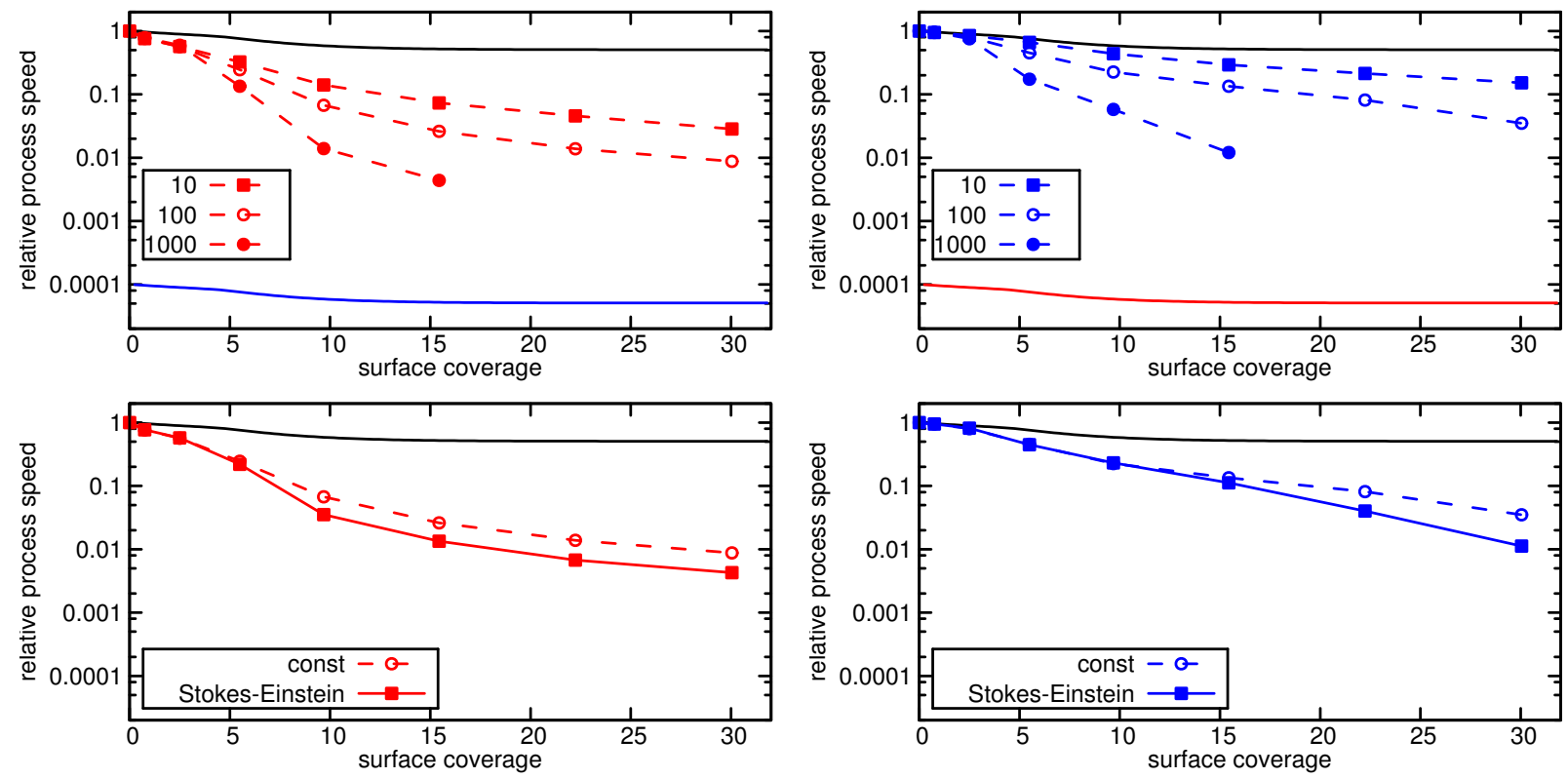

Figure 7: Relative drop coalescence speed $\tau(0) / \tau(\Gamma)$ (on the left) and $\Delta \tau(0) / \Delta \tau(\Gamma)$ (on the right) as a function of the surface coverage $\Gamma$. The effect of viscosity contrast (at constant diffusion coefficients, on the top) and variable diffusion coefficient (at fixed viscosity contrast, on the bottom) are shown. For comparison, the equation of state (black solid curves) and the expected upper diffusion limit (red/blue solid curves) are also shown.

proportional to the viscosity contrast. This indeed explains why the asphaltene-containing oil-water systems can be "stable" emulsions: In real systems, the viscosity contrast between the (otherwise solid) bulk asphaltene and the liquids can be 12 orders of magnitude. Even if the nanometer-thick asphaltane monolayers formed at the liquid-liquid interfaces do not show this enormous contrast in their effective viscosity, the asphaltene surely suppress the flow generated by capillary forces at the interfaces, resulting thus in orders of magnitude longer lifetime of the droplets / slower phase separation. This happens independently from the interfacial tension drop, which is then not responsible for the emulsion formation.

Although the numerical simulations are limited to finite viscosity contrasts, the question what happens in case of highly loaded interfaces $(\omega \approx 20-30)$ in the absence of fluid flow (corresponding to infinite viscosity contrast) still must be answered. First of all, the system becomes diffusion dominated for $|\mathbf{v}| \ll\left|\nabla \cdot \mathbf{J}_{D}\right|$ [i.e. when the velocity field is negligible 
compared to the magnitude of the diffusion fluxes in Equations (5) and (6)]. According to our previous study, the speed of phase separation follows the EOS in this case. In the surfactant-free system, our simulations suggest an $\approx 10^{4}$ faster fluid flow driven process compared to pure diffusion (when $\mathbf{v} \equiv 0$ is set). Therefore, for highly loaded interfaces and infinite viscosity contrast the relative drop coalescence speed in our simulations is expected to be lower than the surface tension reduction $\times$ diffusion/flow process speed contrast in the surfactant-free system. But this refers only to constant diffusion coefficients, and the effect of the thin water layer between the two initial droplets also has to be taken into account. It is reasonable to assume, that an asphaltene monolayer blocks not only the capillary forces, but also the diffusive transport of liquid molecules. The simplest way of including this effect is utilizing the Stokes-Einstein relationship for the diffusion coefficient of the liquids. Consequently, Eq. (5) can be replaced by

$$
\dot{\phi}=\nabla \cdot[d(\phi, \psi) \nabla(\delta \mathcal{F} / \delta \phi)],
$$

where the dimensionless diffusion coefficient $d(\phi, \psi):=h(\phi, 0) / h(\phi, \psi)$ is introduced $[h(\phi, \psi)$ is defined by Eq. (12)]. Note that $d(\phi, \psi)$ is inversely proportional to the local viscosity contrast, and $d(\phi, \psi) \equiv 1$ for $\psi(\mathbf{r}, t) \equiv 0$ (surfactant-free case). $d(\phi, \psi)$ is then expected to reduce further the speed of phase separation as a function of surface coverage. As it is indicated in Fig. 7, the slowdown factor of $2-3$ emerges from this source in case of the bulk viscosity contrast of 100 . The expected overall reduction factor is then surface tension reduction $\times$ diffusion/flow process speed contrast in the surfactant-free system $\times$ diffusion contrast $\times$ factor emerging from the thickening water layer. Our calculations suggest that for highly loaded interfaces the diffusion driven coalescence process does not start (i.e. the oil droplets are not in contact with each other) in $t \sim O\left(10^{6}\right)$, indicating thus at least one order of magnitude extra slowdown from this source. This result also suggests, that even the diffusion controlled system does not follow the interfacial tension drop prescribed by the 
EOS. The overall slowdown with constant diffusion contrast is expected to be then at least 5 orders of magnitude compared to the surfactant-free case, which is further increased by the diffusion contrast, resulting thus in the "stable" emulsion.

\section{Limitations of the numerical method}

To solve Equations (5)-(8) numerically we use an operator-splitting based quasi-spectral semi-implicit method. ${ }^{36}$ The time stepping for a variable is done in the spectral space as:

$$
y^{t+\Delta t}(\mathbf{k}):=y^{t}(\mathbf{k})+\frac{1}{1+\Delta t s_{y}(k)} f_{t}^{t}(\mathbf{k})
$$

where $f_{y}^{t}(\mathbf{k})$ denotes the Fourier component of the right-hand side of the equation $\partial y / \partial t=$ $f_{y}(\ldots)$ at time point $t$, and $s_{y}(k)$ is a suitably chosen splitting operator in the form

$$
s_{y}(k)=S_{1}^{(y)} k^{2}+S_{2}^{(y)} k^{4}+S_{3}^{(y)} k^{6}+\ldots
$$

where $S_{i}^{(y)}$ are non-negative constants determined by the mathematical structure of $f_{y}(\ldots)$. (For details, see Reference 27, for example.) For the Navier-Stokes equation, $S_{1}^{v}$ is proportional to magnitude of the scaled viscosity. The time stepping scheme is nearly unconditionally stable for arbitrary $\Delta t$, which is a huge advantage, but the numerical error of the solution is proportional to the magnitude of $\Delta t s_{y}(k)$. It means, that for increasing viscosity contrast, smaller $\Delta t$ should be chosen in order to control the error, while, coincidentally, longer physical time of the simulation is needed because of the slowdown of the drop coalescence process. These factors then work against each other, making large (>1000) viscosity contrasts practically inaccessible. In order to take all time scales into account simultaneously, including the fluid-flow dominated surfactant-free system to the high-viscosity contrast, slow process speed scenarios, novel numerical methods (such as rigid body techniques based on the dynamic detection of solid-like areas ${ }^{42,43}$ ) must indeed be applied. 


\section{Summary}

In this work the drop coalescence process has been studied qualitatively in water/oil/asphaltene systems. In a scenario, where the interfacial tension drop is limited, emulsion stability cannot be interpreted by the traditional picture of emulsion formation. The formation of emulsions in these systems is rather due to kinetic effects related to the solid-like behavior of the surfactant layer. The phenomenon was addressed by using an convection-diffusion type dynamics utilizing a phenomenological free energy functional. This approach defines two time scales for the system: the time scale of diffusion, and the time scale of fluid flow. In the surfactantfree water-hydrocarbon system fluid flow dominates over diffusion down to the microscale, since capillary forces generate 3 orders of magnitude stronger velocity fields compared to the magnitude of diffusion fluxes. The presence of the surfactant (asphaltenes), however, changes the situation: A rigid asphaltane monolayer forming at the water / oil interface compensates capillarity, i.e. locally suppresses the fluid flow. Consequently, the surfactant layer can be deformed only by diffusion, which is also the only possible transport mode for the liquid molecules to travel through this layer. This phenomenon can be modeled by using a local composition dependent shear viscosity prescribing high contrast between the viscosities of the bulk asphaltene and the bulk liquids. According to our results, the phase separation process / speed of drop coalescence decreases roughly proportional to the viscosity contrast. This effect is limited by the diffusion, which comes into play when the velocity field becomes negligible compared to the diffusion fluxes. Although the maximal expected slowdown of the drop coalescence process is 3 orders of magnitude compared to the surfactant-free case, this applies only in the case of constant diffusion coefficients. Nevertheless, the diffusion coefficients are also expected to be local composition dependent, indicating thus that the asphaltene layer may block the transport of liquid molecules between the two sides of the interface, which apparently leads to a "stable" emulsion. Summarizing, we have a strong theoretical evidence that emulsion formation in water / light hydrocarbon / asphaltene systems can be driven solely by a fluid flow $\rightarrow$ diffusion controlled kinetic transition, where the 
interfacial tension drop is irrelevant.

\section{Acknowledgement}

This work has been supported by the VISTA basic research programme project No. 6359

"Surfactants for water $/ \mathrm{CO}_{2} /$ hydrocarbon emulsions for combined $\mathrm{CO}_{2}$ storage and utilization" of the Norwegian Academy of Science and Letters and the Statoil.

\section{References}

(1) Sjøblom, J. Emulsions and Emulsion Stability: Surfactant Science Series/61; Surfactant Science; Taylor \& Francis, 1996.

(2) Myers, D. Surfactant Science and Technology; Wiley, 2005.

(3) Sarker, D. Pharmaceutical Emulsions: A Drug Developer's Toolbag; Wiley, 2013.

(4) Halpern, D.; Jensen, O. E.; Grotberg, J. B. J. Appl. Physiol. 1998, 85, 333-352.

(5) Hills, B. A. J. Appl. Physiol. 1999, 87, 1567-1583.

(6) Ahearn, G. J. Am. Oil Chem. Soc. 1969, 46, 540A-580A.

(7) Iglauer, S.; Wu, Y.; Shuler, P.; Tang, Y.; III, W. A. G. J. Petrol. Sci. Eng. 2010, 71, $23-29$.

(8) Silset, A. Emulsions (w/o and o/w) of Heavy Crude Oils. Characterization, Stabilization, Destabilization and Produced Water Quality. Ph.D. thesis, Department of Chemical Engineering, Faculty of Natural Science and Technology, Norwegian University of Science and Technology, Trondheim, Norway, 2008.

(9) Tóth, G. I.; Kvamme, B. Phys. Rev. E 2015, 91, 032404.

(10) Tóth, G. I.; Kvamme, B. Phys. Chem. Chem. Phys. 2015, 17, 20259-20273. 
(11) Mikami, Y.; Liang, Y.; Matsuoka, T.; Boek, E. S. Energy \& Fuels 2013, 27, 1838-1845.

(12) Pauchard, V.; Rane, J. P.; Zarkar, S.; Couzis, A.; Banerjee, S. Langmuir 2014, 30, 8381-8390, PMID: 24946262.

(13) Rane, J. P.; Harbottle, D.; Pauchard, V.; Couzis, A.; Banerjee, S. Langmuir 2012, 28, 9986-9995.

(14) Rane, J. P.; Pauchard, V.; Couzis, A.; Banerjee, S. Langmuir 2013, 29, 4750-4759, PMID: 23506138.

(15) Bouriat, P.; El Kerri, N.; Graciaa, A.; Lachaise, J. Langmuir 2004, 20, 7459-7464, PMID: 15323489 .

(16) Pauchard, V.; Sjøblom, J.; Kokal, S.; Bouriat, P.; Dicharry, C.; Müller, H.; al Hajji, A. Energy \&3 Fuels 2009, 23, 1269-1279.

(17) Gompper, G.; Zschocke, S. Phys. Rev. A 1992, 46, 4836-4851.

(18) Theissen, O.; Gompper, G. Eur. Phys. J. B 1999, 11, 91-100.

(19) Teramoto, T.; Yonezawa, F. J. Colloid Interf. Sci. 2001, 235, 329 - 333.

(20) van der Sman, R.; van der Graaf, S. Rheol. Acta 2006, 46, 3-11.

(21) van der Sman, R.; Meinders, M. Computer Physics Communications 2016, 199, 12 21.

(22) Van der Sman, R.; Meinders, M. Pharmaceutical Emulsions: A Drug Developer's Toolbag; Progress in Colloid and Interface Science; CRC Press, 2015; Chapter Mesoscale Lattice Boltzmann Model of Dispersions Stabilized by Surfactants, pp 295-307, 0.

(23) Liu, H.; Zhang, Y. J. Comp. Phys. 2010, 229, 9166 - 9187.

(24) Teng, C.-H.; Chern, I.-L.; Lai, M.-C. Discrete Cont. Dyn. B 2012, 17, 1289 - 1307. 
(25) Li, Y.,; Kim, J., Eur. Phys. J. B 2012, 85, 340.

(26) Engblom, S.; Do-Quang, M.; Amberg, G.; Tornberg, A.-K. Commun. Comput. Phys. 2013, 14, $879-915$.

(27) Lebowitz, J. L.; Percus, J. K. J. Math. Phys. 1963, 4, 116.

(28) Yang, A. J. M.; III, P. D. F.; Gibbs, J. H. The Journal of Chemical Physics 1976, 64, 3732-3747.

(29) Langevin, D. Current Opinion in Colloid \& Interface Science 2015, 20, 92 - 97.

(30) Korbuly, B.; Pusztai, T.; Tóth, G. I.; Henry, H.; Plapp, M.; Gránásy, L. Journal of Crystal Growth 2016,--.

(31) Tóth, G. I.; Pusztai, T.; Gránásy, L. Phys. Rev. B 2015, 92, 184105.

(32) Anderson, D. M.; McFadden, G. B.; Wheeler, A. A. Annu. Rev. Fluid Mech. 1998, 30, $139-165$.

(33) Lowengrub, J.; Truskinovsky, L. Proceedings of the Royal Society of London A: Mathematical, Physical and Engineering Sciences 1998, 454, 2617-2654.

(34) Kim, J.; Lowengrub, J. Intf. Free Bound. 2005, 7, 435 - 466.

(35) Tóth, G. I. Phys. Rev. E 2016, 94, 033114.

(36) Tegze, G.; Bansel, G.; Tóth, G. I.; Pusztai, T.; Fan, Z.; Gránásy, L. J. Comp. Phys. 2009, 228, $1612-1623$.

(37) Tóth, G. I.; Zarifi, M.; Kvamme, B. Phys. Rev. E 2016, 93, 013126.

(38) Price, W. S.; Söderman, O. J. Phys. Chem. A 2000, 104, 5892-5894.

(39) Wang, S.; Xu, J.; Wen, H. Comp. Phys. Commun. 2014, 185, 3069 - 3078. 
(40) Först, P.; Werner, F.; Delgado, A. Rheol. Acta 2000, 39, 566-573.

(41) Ghanavati, M.; Shojaei, M.-J.; S. A., A. R. Energy \& Fuels 2013, 27, 7217-7232.

(42) Iglberger, K.; Thürey, N.; Rüde, U. Computers $\&$ Mathematics with Applications 2008, 55, 1461 - 1468, Mesoscopic Methods in Engineering and Science.

(43) Do-Quang, M.; Amberg, G. Journal of Computational Physics 2008, 227, 1772 - 1789. 